\title{
Efectos de la autofecundación sobre las varianzas aditiva, ambiental y fenotípica, y sobre el valor de la heredabilidad para el rasgo "longitud de la valva" en Argopecten purpuratus, utilizando un modelo animal
}

\author{
Effects of selfing on the additive, environmental and phenotypic variation and on the heritability \\ value for "shell length" in Argopecten purpuratus using an animal model \\ JE Toro ${ }^{a^{*}}$, MP Montoyaa ${ }^{a}$ V Martínez ${ }^{b}$ \\ anstituto de Biología Marina, Facultad de Ciencias, Universidad Austral de Chile, Valdivia, Chile. \\ ${ }^{b}$ Facultad de Ciencias Veterinarias y Pecuarias, Universidad de Chile, Santiago, Chile.
}

\begin{abstract}
SUMMARY
The northern scallop, Argopecten purpuratus, is a hermaphrodite species which has a great economic importance in Chile. Recently, hatchery techniques for juvenile production have been developed for this species, which allows the study the potential effects of selfing in the aquaculture of this species. In the present study we used selfing data obtained in an experiment to establish their effects on the heritability for shell length, by means of stochastic simulations of fullsib family data. Several families were produced using a factorial design (7x7), which allowed the statistical analyses of growth and survival of each family, with variable initial selfing percentage. PCR-based molecular markers (RAPD) developed in a previous study allowed the identification of selfing individuals within each family. The results indicate that heritability values were over-estimate in those families with high percentage of selfing. The selfing percentage was estimated at 6,9 and 12 months of age, observing a significant reduction of it in each of the experimental families. The results obtained lead to conclude that even if the selfing has a negative effect on the development of the northern scallop, this happens only at individual level, affecting the fitness of the individuals which have been produced under that condition. However, those families produced by crossfertilization, which have variable selfing percentages among them, are not significantly affected, because this percentage is reduced gradually with the age of the individuals; therefore, by the time reproductors are selected in a genetic enhancement program, the effect of selfing will not be significant.
\end{abstract}

Palabras clave: autofecundación, heredabilidad, modelo animal.

Key words: selfing, heritability, animal model.

\section{INTRODUCCIÓN}

Argopecten purpuratus, conocido en Chile como el ostión del norte, es una especie de alto interés comercial con 252 centros de cultivo ubicados preferentemente en la zona norte y una producción que aumentó desde 1.182 a 19.426 tons entre 1990 y 2006 (SERNAPESCA 2007), comercializándose principalmente el músculo aductor con o sin la gónada (Taud y Palacios 2003). El cultivo del ostión se realiza en tres fases: captación o producción de semillas, cultivo intermedio y cultivo de engorda, alcanzando la talla comercial entre los 12 y 18 meses. La especie se distribuye naturalmente en las costas del Pacífico desde Corinto, Nicaragua, hasta Valparaíso, Chile, aunque durante los últimos años se han efectuado esfuerzos para iniciar el cultivo de esta especie en aguas más frías del sur de Chile (Taud y Palacios 2003).

El ostión del norte es un molusco bivalvo hermafrodita funcional de fecundación externa que en condiciones naturales inevitablemente genera grados variables de auto-

Aceptado: 24.06.2009.

* Casilla 567, Valdivia, Chile; jtoro@uach.cl fecundación, debido al desove de ambos tipos de gametos en un lapso de tiempo muy corto (Illanes 1988). El desove comienza con los espermatozoides y en un par de minutos desova como hembra, por lo que podrían quedar en el conducto de evacuación de gametos algunos espermatozoides y estos fecundar los ovocitos, generándose así individuos autofecundados. Se ha observado que la autofecundación puede afectar el crecimiento y la sobrevivencia de los individuos, comparados con aquellos de fertilización cruzada (Beaumont y Budd 1983, Gentili y Beaumont 1988). Montoya (2005) desarrolló marcadores moleculares (RAPDs) con un polimorfismo suficiente para poder diferenciar la progenie producida por autofecundación y por fecundación cruzada, y evaluar y cuantificar sus efectos diferenciales a través de su ciclo de vida en condiciones de cultivo. Para el caso de programas de mejoramiento genético, el desconocimiento del porcentaje de individuos autofecundados en las familias y la potencial disminución en la variabilidad genética, puede sesgar seriamente las estimaciones de la variación genética aditiva y la estimación de los efectos de la depresión endogámica.

En la producción de juveniles en laboratorio la condición hermafrodita en ostiones genera un alto porcentaje de individuos autofecundados debido a los reducidos volúmenes 
de agua utilizados (Ibarra y col 1995). La autofecundación es un mecanismo reproductivo que conlleva a una pérdida de variabilidad genética y afecta la capacidad adaptativa de una especie ya que altera el número de loci homocigotos (Tuan y Simões 1998). Los efectos de la autofecundación se han estudiado en moluscos bivalvos como Pecten maximus, observándose una significativa reducción en la tasa de crecimiento en condiciones de cultivo (Beaumont y Budd 1983). Ibarra y col (1995) realizaron un estudio en el cual estimaron el efecto de la autofecundación sobre el crecimiento y la sobrevivencia en larvas de Argopecten circularis, en familias provenientes de autofecundación, por cruzamientos y familias obtenidas por desove en masa. Los autores reportaron que el crecimiento y la sobrevivencia de los individuos autofecundados fue significativamente menor que la de los otros grupos experimentales. Debido a que la heredabilidad de ciertos rasgos podría verse afectada por la autofecundación de forma indirecta al disminuir la potencialidad de determinados caracteres, Ibarra y col (1999) utilizaron un diseño de selección para estimar las heredabilidades en dos rasgos de importancia económica (el peso total y el ancho de la valva) en la especie Argopecten ventricosus. Los autores concluyeron que para el ancho de valva, la variabilidad ambiental es significativamente mayor que la varianza aditiva, lo que indicaría una baja heredabilidad.

El objetivo del presente estudio fue cuantificar los efectos de la autofecundación en A. purpuratus sobre las varianzas aditiva, ambiental y fenotípica y sobre el valor de la heredabilidad para los rasgos tasa de crecimiento y sobrevivencia, mediante simulación estocástica de datos en familias de propios hermanos. Lo anterior reviste importancia ya que al aplicar una determinada intensidad de selección sobre los caracteres cualquier cambio en los componentes de la varianza afectará el valor de la heredabilidad (Toro 1990, Falconer y Mackay 1996, Toro y col 2004 ${ }^{\mathrm{a}}$, Alcapán y col 2007).

\section{MATERIAL Y MÉTODOS}

\section{DISEÑO EXPERIMENTAL}

Se utilizaron ostiones adultos provenientes de bancos naturales de la Bahía Tongoy (IV Región) para generar, mediante un cruzamiento factorial utilizando los desoves individuales de 7 machos y 7 hembras, 49 familias, de las cuales 7 fueron autofecundadas y las 42 producto de fecundación cruzada (Montoya 2005). Debido a su condición hermafrodita, los ostiones desovan primeramente como macho y luego como hembra, por ello al observar los primeros pulsos de espermatozoides se sacaron los ostiones del estanque para posteriormente ser lavados y puestos en recipientes individuales para recuperar los gametos. Después de tres pulsos de espermatozoides los ostiones fueron puestos en nuevos recipientes con agua de mar para el desove como hembras.

\section{AUTOFECUNDACIÓN INICIAL}

Previo a los cruzamientos, se tomaron muestras de los ovocitos de cada una de las hembras, las que se mantuvieron en agua de mar por 12 horas, para luego ser fijadas y establecer mediante recuento directo el porcentaje inicial de ovocitos fecundados por hembra (cuadro 1).

\section{CRECIMIENTO Y SOBREVIVENCIA}

Cada familia se mantuvo en estanques de 200 1, con agua de mar filtrada y esterilizada durante el cultivo larval (Toro y col $2004^{\mathrm{b}}$ ). Las larvas se fijaron a los 30 días en mallas plásticas y se mantuvieron en condiciones de laboratorio durante tres meses, posterior a lo cual los juveniles se trasladaron en bolsas ( $2 \mathrm{~mm}$ de entremalla) etiquetadas con el número de familia correspondiente a "long lines" en la Bahía Hueihue, Chiloé. A los siete meses de edad 300 individuos por familia fueron marcados pegando en caja ejemplar una cinta plástica en la valva conteniendo un código para identificar la familia y al individuo dentro de cada familia (Toro y col $2004^{\text {b }}$ ). Una vez realizado el marcaje y luego de cada medición individual (longitud y peso) a los 6, 9 y 12 meses, las familias se mezclaron y colocaron en linternas japonesas de 10 pisos a una densidad de 50 individuos por piso.

\section{ANÁLISIS ESTADÍSTICO FENOTÍPICO}

El efecto de los valores iniciales de autofecundación de cada una de las hembras sobre el peso vivo (g) y longitud de la valva $(\mathrm{mm})$ de su progenie fue analizado mediante un modelo de regresión lineal simple con el paquete estadístico SAS (versión 9.1) (SAS Institute Inc., Cary, NC, USA). Se determinó además la productividad y la ganancia tanto del peso vivo como de la longitud de la valva. La productividad se estimó como el incremento en $\mathrm{mg}$ o $\mathrm{mm}$ respectivamente, en relación a los 90 días entre cada muestreo y la ganancia como el incremento en longitud de la valva en cada muestreo. Se aplicó un análisis de varianza de una vía para determinar si el tipo de fecundación (cruzada o autofecundación) afectaba la productividad y ganancia.

Cuadro 1. Porcentaje inicial de ovocitos autofecundados (\%) a las 12 horas postdesove para cada hembra en A. purpuratus.

Initial percentage $(\%)$ of selfertilized oocytes at $12 \mathrm{~h}$ after spawning for each female of A. purpuratus.

\begin{tabular}{cc}
\hline Hembra & \% de ovocitos autofecundados \\
\hline 1 & 14,16 \\
2 & 45,76 \\
3 & 14,50 \\
4 & 18,03 \\
5 & 17,90 \\
6 & 23,20 \\
7 & 9,96 \\
Promedio & 20,50 \\
\hline
\end{tabular}


Cuadro 2. Análisis de varianza para longitud de la valva, peso vivo, la productividad y ganancia de ambos rasgos, en las familias de cruzamiento, considerando la autofecundación, en distintas etapas del ciclo de vida de A. purpuratus.

Analysis of variance for shell length, live weight, productivity and gain of both traits, among families produced by crossing, considering selfing at different stages of the life cycle of A. purpuratus.

\begin{tabular}{|c|c|c|c|c|c|}
\hline & Fuente & GL & $\mathrm{CM}$ & $\mathrm{F}$ & $\mathrm{P}$ \\
\hline \multicolumn{6}{|l|}{6 Meses } \\
\hline \multirow[t]{2}{*}{ Longitud valva } & Modelo & 1 & 6,60 & 0,316 & $\mathrm{~ns}$ \\
\hline & Error & 1.499 & 20,89 & & \\
\hline \multirow[t]{2}{*}{ Productividad } & Modelo & 1 & $8.910 .306,01$ & 30,180 & * \\
\hline & Error & 1.499 & $295.242,94$ & & \\
\hline \multirow[t]{2}{*}{ Ganancia } & Modelo & 1 & 0,02 & 15,482 & * \\
\hline & Error & 1.499 & 0,00 & & \\
\hline \multicolumn{6}{|l|}{9 Meses } \\
\hline \multirow[t]{2}{*}{ Longitud valva } & Modelo & 1 & 139,37 & 5,174 & * \\
\hline & Error & 1.499 & 26,90 & & \\
\hline \multirow[t]{2}{*}{ Productividad } & Modelo & 1 & $2.009 .745,20$ & 4,896 & * \\
\hline & Error & 1.499 & $410.447,90$ & & \\
\hline \multirow[t]{2}{*}{ Ganancia } & Modelo & 1 & 0,00 & 1,650 & $*$ \\
\hline & Error & 1.499 & 0,00 & & \\
\hline \multirow{2}{*}{ Peso vivo } & Modelo & 1 & 148,38 & 5,8 & * \\
\hline & Error & 1.499 & 25,41 & & \\
\hline \multirow[t]{2}{*}{ Productividad } & Modelo & 1 & $2.832 .118,80$ & 11,486 & $*$ \\
\hline & Error & 1.499 & $239.077,90$ & & \\
\hline \multicolumn{6}{|l|}{12 Meses } \\
\hline \multirow[t]{2}{*}{ Longitud valva } & Modelo & 1 & 35,80 & 1,077 & $\mathrm{~ns}$ \\
\hline & Error & 1.499 & 33,23 & & \\
\hline \multirow[t]{2}{*}{ Ganancia } & Modelo & 1 & 0,00 & 2,636 & $\mathrm{~ns}$ \\
\hline & Error & 1.499 & 0,00 & & \\
\hline \multirow{2}{*}{ Peso vivo } & Modelo & 1 & 0,02 & 0,000 & $\mathrm{~ns}$ \\
\hline & Error & 1.499 & 99,73 & & \\
\hline \multirow[t]{2}{*}{ Productividad } & Modelo & 1 & $27.868,71$ & 0,033 & $\mathrm{~ns}$ \\
\hline & Error & 1.499 & $835.161,92$ & & \\
\hline
\end{tabular}

GL: Grados de libertad; CM: cuadrado medio; P: probabilidad; *: significativo $\mathrm{P}<0,05$; ns: no significativo.

Se determinó la tasa de mortalidad por familia a los 9 y 12 meses, con el fin de llevar a cabo un análisis de varianza de una vía con datos transformados a arcoseno, para determinar si los porcentajes iniciales de autofecundación fueron distintos entre las familias de fecundación cruzada. La identificación de cada uno de los individuos experimentales autofecundados se llevó a cabo utilizando marcadores moleculares RAPDs (Montoya 2005, Martínez 2007).

\section{HEREDABILIDAD Y GRADO DE AUTOFECUNDACIÓN}

Para determinar el comportamiento de la heredabilidad de los rasgos, peso vivo y longitud de la valva en los individuos experimentales, se llevó a cabo una simulación estocástica con datos de familias de propios hermanos, utilizando el siguiente modelo animal desarrollado por Martínez (2008): $\mathrm{Y}=\mathrm{Xb}+\mathrm{Zu}+\mathrm{e}$, donde $\mathrm{Y}$ es el vector de observaciones, $b$ es el vector de efectos fijos (que incluyen el promedio poblacional), u es el vector de efectos genéticos aditivos y e es el vector de efectos residuales ambientales. Se realizaron al menos 1000 réplicas para cada simulación en el software estadístico SAS. X y Z son matrices de incidencia conocidas, que permiten relacionar los vectores $\mathrm{b}$ y a con el vector de las observaciones. Este modelo permite predecir el comportamiento de los parámetros genéticos de un carácter, que potencialmente pudiera ser escogido para selección, a través del análisis de parentesco para obtener los estimados de estos parámetros en la siguiente generación. Se trata de un modelo aditivo, donde no hay dominancia. Para este propósito, la variable utilizada fue la heredabilidad con valores de $0,05,0,1,0,2,0,3,0,4$ y 0,5 que se contrastaron sobre un grupo control (sin autofecundación) y un grupo con autofecundación, observándose el comportamiento de la heredabilidad estimada, luego de dos generaciones, a partir de una población de 500 individuos (Martínez y Di Giovanni 2007). La autofecundación en el grupo que la presenta se simuló en relación a la distribución de autofecundados al inicio del ciclo (una distribución normal con promedio $20 \% \pm 12$ ). Los resultados obtenidos que incluyen valores de varianza genética, varianza ambiental y varianza fenotípica, además de la heredabilidad estimada, fueron analizados con el paquete estadístico SAS, mediante análisis de varianza, usando el siguiente modelo estadístico: $\mathrm{y}_{\mathrm{ij}}=\mu+\mathrm{f}_{\mathrm{i}}+\mathrm{e}_{\mathrm{ij}}$, donde, $\mathrm{y}_{\mathrm{ij}}$ es el valor de la 
Cuadro 3. Análisis de varianza, para el efecto de la autofecundación sobre la mortalidad, en familias de cruzamiento a los 9 y 12 meses de edad en A. purpuratus.

Analysis of variance for the effect of selfing on the mortality among families produced by crossing at 9 and 12 months of age in $A$. purpuratus.

\begin{tabular}{llcccc}
\hline 9 Meses & Fuente & GL & CM & F & P \\
\hline \multirow{4}{*}{12 Meses } & Modelo & 1 & 926,50 & 78,84 & $*$ \\
& Error & 3.992 & 12,20 & & \\
& Errodelo & 1 & 68,29 & 1,49 & ns \\
\hline
\end{tabular}

GL: Grados de libertad; CM: cuadrado medio; P: probabilidad; *: significativo $\mathrm{P}<0,05$; ns: no significativo.

Cuadro 4. Porcentaje de individuos autofecundados (\%) dentro de las familias de cruzamiento por estadio de desarrollo en $A$. purpuratus.

Percentage of selfing (\%) among families produced by crossing at each stage of development in A. purpuratus.

\begin{tabular}{cccccc}
\hline Familia & $\begin{array}{c}\text { Hembra } \\
\%\end{array}$ & $\begin{array}{c}\text { Inicial } \\
\%\end{array}$ & $\begin{array}{c}\text { Larvas } \\
\%\end{array}$ & $\begin{array}{c}\text { Juvenil } \\
\%\end{array}$ & $\begin{array}{c}\text { Adultos } \\
\%\end{array}$ \\
\hline F08 & 2 & 45,8 & 18,7 & 4,7 & 2,20 \\
F10 & 2 & 45,8 & 20,0 & 6,6 & 0,00 \\
F15 & 3 & 14,9 & 8,3 & 0,0 & 0,00 \\
F16 & 3 & 14,9 & 14,2 & 7,2 & 5,13 \\
F19 & 3 & 14,9 & 4,0 & 0,0 & 0,00 \\
F20 & 3 & 14,9 & 14,2 & 8,3 & 0,00 \\
F28 & 5 & 17,9 & 7,6 & 0,0 & 0,00 \\
F36 & 6 & 23,2 & 0,0 & 0,0 & 2,56 \\
F38 & 6 & 23,2 & 14,2 & 5,0 & 3,23 \\
F39 & 6 & 23,2 & 0,0 & 0,0 & 0,00 \\
Promedio & & 23,8 & 7,2 & 4,9 & 1,57 \\
\hline
\end{tabular}

heredabilidad o componente de varianza de la j-ésima réplica, $\mathrm{f}_{\mathrm{i}}$ es el efecto fijo de la autofertilización o control $\mathrm{y} \mathrm{e}_{\mathrm{ij}}$ son los efectos residuales aleatorios. Esto permitió establecer si las diferencias entre la heredabilidad inicial y la estimada obtenidas entre ambos grupos fueron o no significativas. Los análisis de las varianzas genética, ambiental y fenotípica nos permitieron determinar cuál de estos tres componentes es el responsable del cambio en las heredabilidades estimadas.

\section{RESULTADOS}

\section{CRECIMIENTO Y SOBREVIVENCIA}

La obtención de familias mediante el diseño factorial permitió llegar al término del experimento con 25 familias, cuatro de las cuales fueron representantes de las familias autofecundadas. El comportamiento de la longitud de la valva $(\mathrm{mm})$ durante los tres estadios de desarrollo (larva, semilla y juvenil) mostró una disminución a medida que aumentaron los porcentajes de autofecundación. Sin embargo, esta disminución sólo fue significativa a los 6 meses (cuadro 2), además, el comportamiento de la productividad a la misma edad es también significativo con una disminución que se correlaciona con el aumento en los porcentajes de autofecundación. Similar comportamiento tuvo la ganancia de la longitud de la valva, sin embargo, este fue significativo a los 6 y 9 meses de edad (cuadro 2). El carácter peso vivo del ostión tiende a disminuir con el aumento de la autofecundación, sin embargo, este efecto sólo fue significativo a los 9 meses de edad (cuadro 2). Este mismo comportamiento se observa para la productividad del carácter peso vivo, para el cual el efecto que produce un aumento en el porcentaje de autofecundación sólo fue

Cuadro 5. Análisis de varianza para longitud de la valva $(\mathrm{mm})$ y peso vivo $(\mathrm{g})$ a los 9 y 12 meses de edad, comparando familias autofecundadas versus de cruzamiento en $A$. purpuratus.

Analysis of variance for shell length $(\mathrm{mm})$ and live weight $(\mathrm{g})$ at 9 and 12 months of age, comparing families produced by selfing and crossing in A. purpuratus

\begin{tabular}{llcccc}
\hline 9 meses & Fuente & GL & CM & F & P \\
\hline Talla & Tipo & 1 & 940.858 & 18,010 & $*$ \\
& Error & 3.062 & 52.242 & & \\
\multirow{2}{*}{ Peso } & Tipo & 1 & 294.458 & 8,846 & $*$ \\
& Error & 3.062 & 33.288 &
\end{tabular}

12 meses

\begin{tabular}{cccccc} 
Talla & Tipo & 1 & 471.525 & 11,821 & $*$ \\
& Error & 1.872 & 39.887 & & \\
Peso & Tipo & 1 & 325.885 & 2,571 & $\mathrm{~ns}$ \\
& Error & 1.872 & 126.733 & & \\
\hline
\end{tabular}

GL: Grados de libertad; CM: cuadrado medio; P: probabilidad; *: significativo $\mathrm{P}<0,05$; ns: no significativo. 
Cuadro 6. Media de las varianzas aditiva, ambiental, fenotípica y de la heredabilidad esperada \pm DS, en familias con y sin autofecundación utilizando distintos valores de heredabilidad en A. purpuratus.

Mean of the additive, environmental and phenotypic variances and the expected heritabilities \pm SD in families with and without selfing, using different heritability values in A. purpuratus.

\begin{tabular}{|c|c|c|c|c|c|c|c|c|c|}
\hline \multirow{3}{*}{$\begin{array}{c}\text { Heredabilidad } \\
0,05\end{array}$} & \multirow{3}{*}{$\begin{array}{c}\text { Autofecundación } \\
\text { no }\end{array}$} & \multirow{2}{*}{\multicolumn{4}{|c|}{$\begin{array}{c}\text { Varianza } \\
\text { Ambiental } \\
\text { media } \pm \text { SD }\end{array}$}} & \multirow{2}{*}{\multicolumn{2}{|c|}{$\begin{array}{c}\text { Fenotípica } \\
\text { media } \pm \text { SD }\end{array}$}} & \multirow{2}{*}{\multicolumn{2}{|c|}{$\begin{array}{l}\text { Heredabilidad } \\
\text { esperada } \\
\text { media } \pm \text { SD }\end{array}$}} \\
\hline & & & & & & & & & \\
\hline & & 0,051 & 0,028 & 0,948 & 0,045 & 0,999 & 0,038 & 0,051 & 0,028 \\
\hline \multirow{3}{*}{0,1} & sí & 0,094 & 0,028 & 0,931 & 0,036 & 1,025 & 0,033 & 0,091 & 0,027 \\
\hline & no & 0,101 & 0,036 & 0,898 & 0,047 & 1,000 & 0,039 & 0,101 & 0,035 \\
\hline & sí & 0,185 & 0,046 & 0,866 & 0,039 & 1,051 & 0,037 & 0,175 & 0,040 \\
\hline \multirow[t]{2}{*}{0,2} & no & 0,201 & 0,051 & 0,797 & 0,048 & 0,999 & 0,042 & 0,201 & 0,047 \\
\hline & sí & 0,339 & 0,062 & 0,749 & 0,042 & 1,088 & 0,044 & 0,310 & 0,048 \\
\hline \multirow[t]{2}{*}{0,3} & no & 0,300 & 0,063 & 0,697 & 0,048 & 0,998 & 0,045 & 0,299 & 0,055 \\
\hline & sí & 0,465 & 0,071 & 0,648 & 0,041 & 1,113 & 0,051 & 0,416 & 0,049 \\
\hline \multirow[t]{2}{*}{0,4} & no & 0,401 & 0,073 & 0,597 & 0,049 & 0,999 & 0,049 & 0,400 & 0,059 \\
\hline & sí & 0,565 & 0,073 & 0,562 & 0,038 & 1,128 & 0,055 & 0,499 & 0,046 \\
\hline \multirow[t]{2}{*}{0,5} & no & 0,500 & 0,080 & 0,498 & 0,049 & 0,998 & 0,051 & 0,498 & 0,061 \\
\hline & sí & 0,631 & 0,068 & 0,494 & 0,034 & 1,125 & 0,053 & 0,559 & 0,040 \\
\hline
\end{tabular}

significativo a los 9 meses de edad (cuadro 2). En cuanto al efecto de la autofecundación sobre la mortalidad, en el cuadro 3 se observa que éste efecto es significativo a los 9 meses de edad, pero no a los 12 meses de edad.

En el cuadro 4, se muestran los porcentajes de autofecundación en 10 familias y para cada una de las cuatro hembras progenitoras. Estos datos nos permiten observar cómo disminuyen significativamente $(\mathrm{P}<0,05)$ los porcentajes de autofecundación en las distintas etapas del ciclo de vida.

En el cuadro 5 se muestran los análisis de varianza para la longitud de la valva y el peso vivo del ostión, comparando el comportamiento de dichos caracteres en familias autofecundadas y de cruzamiento. Para ambos rasgos se observa una diferencia significativa a favor de los individuos provenientes de cruzamiento, con la excepción del peso vivo a los 12 meses. La tasa de sobrevivencia de las familias autofecundadas, comparada con la de las familias provenientes de cruzamiento a través de un análisis de varianza, indica que la sobrevivencia se ve significativamente $(\mathrm{P}<0,05)$ afectada por la autofecundación.

\section{HEREDABILIDAD Y GRADO DE AUTOFECUNDACIÓN}

Comparando los resultados obtenidos en ambos grupos (control y con autofecundación), se observa que la autofecundación presenta un efecto significativo sobre el valor de la heredabilidad haciendo que esta sea sobrestimada. Tal efecto está dado por el hecho de que hay una redistribución de las varianzas (debido a que no se incluyen las covarianzas entre autofecundados y propios hermanos), que hace que varíe la varianza genética aditiva. En la figura 1 se muestran los histogramas obtenidos al graficar el comportamiento de las heredabilidades estimadas, considerando ambos grupos. Se observa claramente que en el grupo con autofecundación, la heredabilidad presenta un sesgo hacia la derecha, es decir, que en presencia de autofecundación la heredabilidad del carácter tendería a ser más alta y en el caso del grupo control se observa que esta se mantendría similar a la de la población base. En la figura 2a se compara directamente lo que ocurre con un valor de heredabilidad de 0,05 , en el grupo autofecundado esta aumenta casi al doble llegando en promedio a 0,09 . Lo mismo se puede observar en los demás histogramas, en donde se grafican los efectos para las heredabilidades $0,1,0,2,0,3,0,4$ y 0,5 . en ambos grupos.

\section{ANÁLISIS ESTADÍSTICO DE LOS DATOS DE SIMULACIÓN}

En el cuadro 6 se observa el efecto directo que tiene la autofecundación sobre la media de las varianzas aditiva, ambiental, fenotípica y de la heredabilidad estimada. Se observó un aumento de la varianza aditiva con un consecuente aumento en la varianza fenotípica y en la heredabilidad observada. La diferencia entre ambos valores, entre el grupo control y el grupo con autofecundación, se incrementa levemente, mientras mayor es el valor de la heredabilidad. En el cuadro 7 se presentan los resultados de los análisis estadísticos para medir la significancia de dichas diferencias. Estas diferencias fueron significativas, exceptuando el caso de la varianza ambiental, frente a una heredabilidad de 0,5 . 

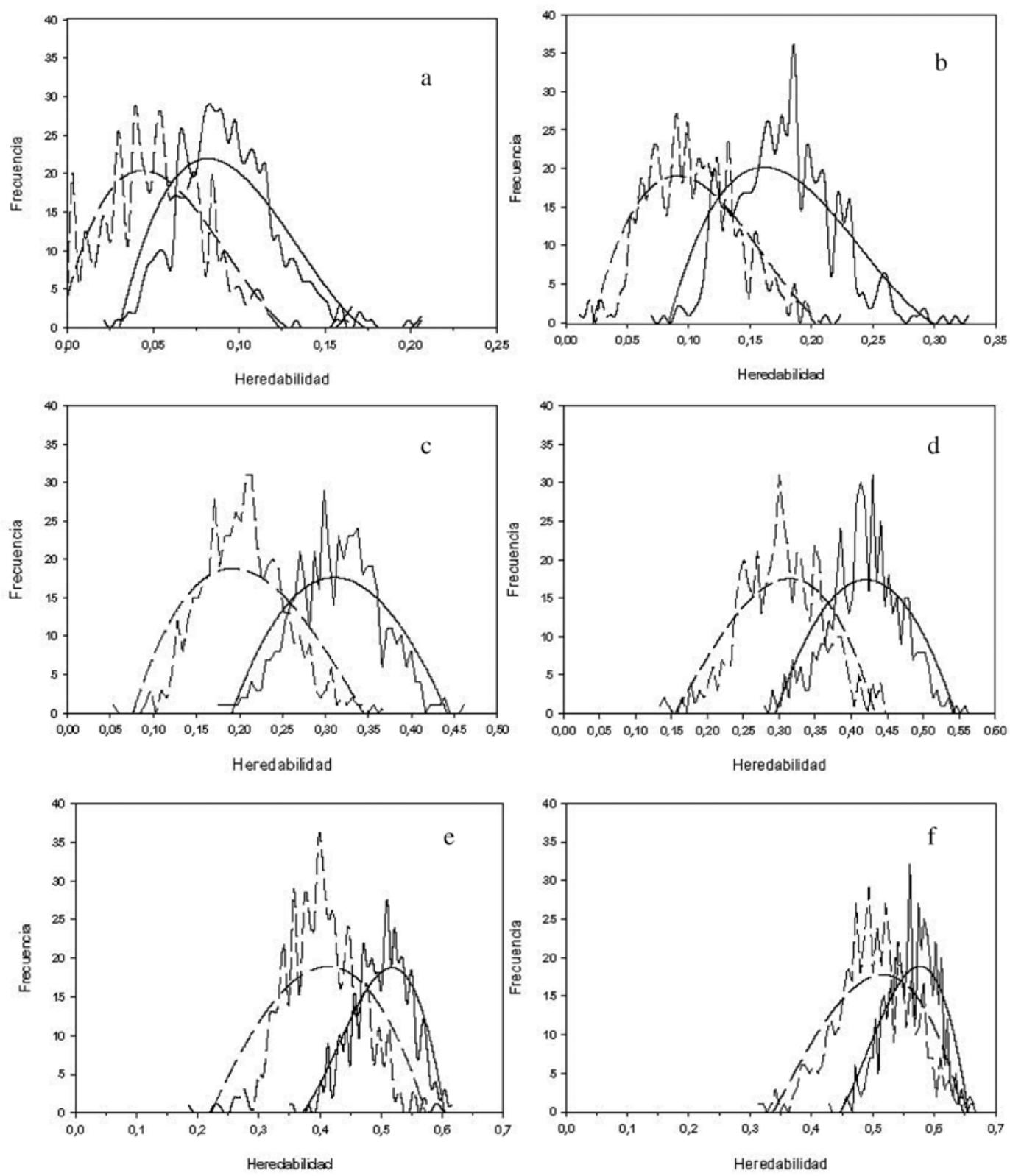

Figura 1. Comportamiento de la heredabilidad observada, con valores iniciales para la heredabilidad de a) 0,05 , b) 0,1 , c) 0,2 , d) 0,3 , e) 0,4 y f) 0,5 , con (—) y sin (--- ) presencia de autofecundación en A. purpuratus.

Behaviour of the expected heritability, using initial heritability values of a) 0.05, b) 0.1, c) 0.2, d) 0.3 , e) 0.4 and f) 0.5 , with $\sin (\longrightarrow$ ) and without selfing (----) in A. purpuratus.

\section{DISCUSIÓN}

La autofecundación ha sido estudiada en moluscos hermafroditas funcionales, como caracoles y ostiones (Tuan y Simões 1998, Ibarra y col 1999), y en la mayoría de los casos se ha establecido que los organismos originados de esta manera poseen un menor fitness (adecuación biológica) que su contraparte producto de cruzamiento (Beaumont y Budd 1983, Gentili y Beaumont 1988, Falconer y Mackay 1996). Teóricamente, en la naturaleza la autofecundación en organismos hermafroditas no ocurriría con tanta fre- cuencia como en condiciones de cultivo artificial (Ibarra y col 1995), en donde los reducidos volúmenes de agua utilizados afectarían el porcentaje de autofecundación.

$\mathrm{Al}$ analizar los efectos de la autofecundación sobre distintos caracteres fenotípicos descritos en la literatura (Beaumont y Budd 1983, Ibarra y col 1995, Tuan y Simões 1998, Winkler y Estévez 2003) se observa que la edad de los organismos al momento de ser evaluados tiene un efecto significativo. Winkler y Estévez (2003) estudiaron los efectos de la autofecundación en los primeros estadios de desarrollo de A. purpuratus y concluyen que no existe 
Cuadro 7. Análisis de varianza para la varianza aditiva (VA), ambiental (VE), fenotípica (VF) y heredabilidad estimada (h²e) para determinar el efecto de la autofecundación, usando distintos valores de heredabilidad $\left(\mathrm{h}^{2}\right)$.

in A. purpuratus.

Analysis of variance for the additive (VA), environmental (VE), phenotypic (VF) variances and the expected heritability $\left(\mathrm{h}^{2} \mathrm{e}\right)$ to determine the effect of selfing using different heritability $\left(\mathrm{h}^{2}\right)$ values

\begin{tabular}{|c|c|c|c|c|c|c|c|c|c|c|c|c|c|}
\hline $\mathrm{h}^{2}$ & 0,05 & & VA & & & VE & & & $\mathrm{VF}$ & & & $h^{2} E$ & \\
\hline Fuente & GL & $\mathrm{SC}$ & $\mathrm{F}$ & $\mathrm{P}$ & $\mathrm{SC}$ & $\mathrm{F}$ & $\mathrm{P}$ & $\mathrm{SC}$ & $\mathrm{F}$ & $\mathrm{P}$ & $\mathrm{SC}$ & $\mathrm{F}$ & $\mathrm{P}$ \\
\hline Modelo & 1 & 0,462 & 551 & $*$ & 0,070 & 41,63 & $*$ & 0,171 & 129,09 & $*$ & 0,408 & 523,64 & $*$ \\
\hline Error & 998 & 0,836 & & & 1,691 & & & 1,323 & & & 0,778 & & \\
\hline
\end{tabular}

\begin{tabular}{|c|c|c|c|c|c|c|c|c|c|c|c|c|c|}
\hline $\mathrm{h}^{2}$ & 0,1 & & VA & & & VE & & & $\mathrm{VF}$ & & & $h^{2} E$ & \\
\hline Fuente & GL & $\mathrm{SC}$ & $\mathrm{F}$ & $\mathrm{P}$ & $\mathrm{SC}$ & $\mathrm{F}$ & $\mathrm{P}$ & $\mathrm{SC}$ & $\mathrm{F}$ & $\mathrm{P}$ & $\mathrm{SC}$ & $\mathrm{F}$ & $\mathrm{P}$ \\
\hline Modelo & 1 & 1,732 & 992,87 & $*$ & 0,258 & 136,01 & $*$ & 0,651 & 441,84 & $*$ & 1,360 & 929,74 & $*$ \\
\hline Error & 998 & 1,741 & & & 1,898 & & & 1,472 & & & 1,460 & & \\
\hline
\end{tabular}

\begin{tabular}{|c|c|c|c|c|c|c|c|c|c|c|c|c|c|}
\hline $\mathrm{h}^{2}$ & 0,2 & & VA & & & VE & & & VF & & & $h^{2} E$ & \\
\hline Fuente & GL & $\mathrm{SC}$ & $\mathrm{F}$ & $\mathrm{P}$ & $\mathrm{SC}$ & $\mathrm{F}$ & $\mathrm{P}$ & $\mathrm{SC}$ & $\mathrm{F}$ & $\mathrm{P}$ & $\mathrm{SC}$ & $\mathrm{F}$ & $\mathrm{P}$ \\
\hline Modelo & 1 & 4,741 & 1427,80 & $*$ & 0,582 & 279,21 & $*$ & 2,000 & $1.050,00$ & $*$ & 2,989 & $1.274,84$ & $*$ \\
\hline Error & 998 & 3,314 & & & 2,081 & & & 1,901 & & & 2,34 & & \\
\hline
\end{tabular}

\begin{tabular}{|c|c|c|c|c|c|c|c|c|c|c|c|c|c|}
\hline $\mathrm{h}^{2}$ & 0,3 & & VA & & & VE & & & VF & & & $h^{2} E$ & \\
\hline Fuente & GL & $\mathrm{SC}$ & $\mathrm{F}$ & $\mathrm{P}$ & $\mathrm{SC}$ & $\mathrm{F}$ & $\mathrm{P}$ & $\mathrm{SC}$ & $\mathrm{F}$ & $\mathrm{P}$ & $\mathrm{SC}$ & $\mathrm{F}$ & $\mathrm{P}$ \\
\hline Modelo & 1 & 6,749 & 1466,60 & $*$ & 0,605 & 296,39 & $*$ & 3,311 & $1.386,13$ & $*$ & 3,371 & $1.203,14$ & * \\
\hline
\end{tabular}

\begin{tabular}{|c|c|c|c|c|c|c|c|c|c|c|c|c|c|}
\hline $\mathrm{h}^{2}$ & 0,4 & & VA & & & VE & & & VF & & & $h^{2} E$ & \\
\hline Fuente & GL & $\mathrm{SC}$ & $\mathrm{F}$ & $\mathrm{P}$ & $\mathrm{SC}$ & $\mathrm{F}$ & $\mathrm{P}$ & $\mathrm{SC}$ & $\mathrm{F}$ & $\mathrm{P}$ & $\mathrm{SC}$ & $\mathrm{F}$ & $\mathrm{P}$ \\
\hline Modelo & 1 & 6,729 & 1240,25 & $*$ & 0,303 & 155,98 & $*$ & 4,174 & $1.523,35$ & $*$ & 2,477 & 865,22 & $*$ \\
\hline Error & 998 & 5,415 & & & 1,943 & & & 2,734 & & & 2,857 & & \\
\hline
\end{tabular}

\begin{tabular}{|c|c|c|c|c|c|c|c|c|c|c|c|c|c|}
\hline $\mathrm{h}^{2}$ & 0,5 & & VA & & & VE & & & VF & & & $h^{2} E$ & \\
\hline Fuente & GL & $\mathrm{SC}$ & $\mathrm{F}$ & $\mathrm{P}$ & $\mathrm{SC}$ & $\mathrm{F}$ & $\mathrm{P}$ & $\mathrm{SC}$ & $\mathrm{F}$ & $\mathrm{P}$ & $\mathrm{SC}$ & $\mathrm{F}$ & $\mathrm{P}$ \\
\hline Modelo & 1 & 4,289 & 771,45 & $*$ & 0,003 & 2,17 & ns & 4,034 & $1.457,94$ & $*$ & 0,918 & 340,58 & * \\
\hline Error & 998 & 5,549 & & & 1,793 & & & 2,761 & & & 2,690 & & \\
\hline
\end{tabular}

GL: Grados de libertad; SC: suma de cuadrados; P: probabilidad*: significativo P $<0,05$; ns: no significativo. 
diferencia en el crecimiento y la sobrevivencia de larvas y juveniles al comparar familias producidas por cruzamiento versus las producidas por autofecundación. Sus conclusiones concuerdan con los resultados obtenidos en el presente estudio ya que hasta la etapa de juvenil de seis meses no se observaron grandes diferencias en longitud de la valva y peso vivo entre las familias de autofecundados y las provenientes de cruzamientos. En el caso de Pecten maximus (Beaumont y Budd 1983), la autofecundación afectó sólo la tasa de crecimiento de las larvas veliger (18 días de edad) en todos los cultivos de su diseño experimental. Por ello resulta interesante evaluar el comportamiento de los individuos en sus distintas etapas del ciclo de vida.

A través de análisis de genética cuantitativa se observó que la heredabilidad se ve afectada por la autofecundación provocando un incremento en los valores estimados de esta. Este incremento en el valor de la heredabilidad tendría su origen por el reordenamiento que provoca la varianza, como consecuencia de un cambio en las frecuencias genotípicas, debido principalmente a que entre cada generación existe un factor de endogamia que debe ser considerado, sobre todo cuando se trata de una especie hermafrodita en donde la autofecundación ocurre naturalmente. Sin embargo, hay que señalar que este factor estaría cambiando las frecuencias genotípicas y no las frecuencias génicas (Falconer y Mackay 1996). En este estudio la población base estaba bajo el supuesto de que se trata de un modelo aditivo sin dominancia, en el cual se conocen las frecuencias genotípicas. Los valores de la heredabilidad estimada en el grupo control sin autofecundación fueron en todos los casos similares a los iniciales, es decir, que la siguiente generación tendría un comportamiento similar a la población base teniendo en cuenta los supuestos del modelo.

La mayor parte de los estudios en organismos hermafroditas que se han realizado hasta ahora han cuantificado diferencias entre las familias generadas por autofecundación y las producidas por cruzamiento. Estas diferencias se centran en los caracteres fenotípicos de dichas familias, analizándolas a través de análisis estadísticos. En algunos trabajos como los realizados por Beaumont y Budd (1983) en $P$. maximus, Ibarra y col (1995) en A. circularis, y otros estudios en especies como Crassotrea virginica y Mytilus edulis, se ha visto que la autofecundación efectivamente afecta negativamente el fitness de estas especies (Gentili y Beaumont 1988). Sin embargo, existen muy poco trabajos destinados a encontrar o a identificar a los individuos autofecundados dentro de las familias que fueron originadas por cruzamiento y sus potenciales efectos sobre distintos caracteres en dichas familias. Vernon y col (1995) utilizando marcadores RAPDs llevaron a cabo un estudio destinado a identificar a los individuos producidos por cruzamiento dentro de familias del caracol (también hermafrodita funcional) Biomphalaria glabrata. Los autores lograron identificar los patrones de bandas que serían propios de cada uno de los individuos provenientes de cruzamiento. En el presente estudio, la técnica de RAPDs permitió la identificación de los individuos autofecundados con bastante precisión (Vernon y col 1995, Rego y col 2002, Feráld 2002, Liu y Cordes 2004, Montoya 2005).

El análisis estadístico para examinar los efectos de la autofecundación directamente sobre el peso y talla del ostión permitió concluir que el efecto mayor ocurre a la edad de 9 meses. Este efecto negativo implica que mientras mayores son los niveles de autofecundación menor es el crecimiento de los ostiones y a su vez mayor la mortalidad de los mismos. La autofecundación provoca un aumento en la endogamia con efectos como reducción de la heterocigosidad y depresión del fitness (Falconer y Mackay 1996). En este contexto, existen trabajos que han determinado la relación que hay entre la heterocigosidad y el fitness, medido a través del comportamiento que tiene en caracteres como sobrevivencia, fecundidad y crecimiento (Koehn y Shumway 1982, Rodhouse y col 1986, Blot y Thiriot-Quievreux 1988, Alvarez y col 1989, Toro y col 1996, Toro y col 1998). Todos estos autores obtuvieron evidencia que indica una correlación positiva entre la heterocigosidad multilocus (HML) y la tasa de sobrevivencia, la producción de gametos y la tasa de crecimiento, lo que permite concluir que aquellos individuos con una mayor aptitud biológica son consecuencia de una mayor HML (David 1998, Toro y col 1998).

Los resultados del presente estudio permiten concluir que los efectos directos de la autofecundación en las familias producidas por cruzamiento, como reducción en la tasa de crecimiento y sobrevivencia, solo fueron evidentes en la etapa juvenil del ostión del norte. Lo anterior debido a que se observó una disminución significativa de individuos autofecundados dentro de las familias de cruzamiento a medida que se avanza en el ciclo de vida del ostión. Además, la presencia de individuos autofecundados produce un cambio significativo en los valores de la heredabilidad, haciendo que estos sean sobrestimados para los rasgos longitud de la valva y peso vivo en $A$. purpuratus.

\section{RESUMEN}

El ostión del norte, Argopecten purpuratus, es una especie hermafrodita, de gran interés comercial en Chile. Recientemente se han desarrollado técnicas para la producción de juveniles en laboratorio, lo cual permite utilizar a esta especie para estudios de los potenciales efectos que tendría la autofecundación en el cultivo y crecimiento del ostión del norte. En el presente trabajo se utilizan datos de autofecundación obtenidos experimentalmente, para establecer los efectos sobre la heredabilidad de la tasa de crecimiento, mediante simulación estocástica de datos de familias de propios hermanos. Se generaron familias mediante una matriz factorial de cruzamientos $(7 \times 7)$, lo que permitió realizar un análisis estadístico de datos de longitud de valva, peso vivo y sobrevivencia, de cada una de las familias, con distintos porcentajes de autofecundación inicial. Por otra parte, se desarrollaron marcadores moleculares basados en la reacción PCR (RAPDs), que permitieron identificar a los individuos autofecundados dentro de cada una de las familias producidas. Se estimó el porcentaje de autofecundados a los 6,9 y 12 meses de edad, 
observando una reducción significativa de éstos en cada una de las familias experimentales. Los resultados permiten concluir que si bien la autofecundación afecta negativamente el desarrollo del ostión del norte, este efecto se refleja directamente en forma individual, afectando la adecuación biológica (fitness) de los individuos producidos bajo dicha condición. Sin embargo, las familias generadas por cruzamiento, que tienen porcentajes de autofecundación variables dentro de ellas, no se ven significativamente afectadas, ya que este efecto disminuye gradualmente con la edad y por lo tanto en el momento de hacer la selección para el programa de mejoramiento genético el efecto de la autofecundación no sería significativo.

\section{AGRADECIMIENTOS}

Los autores agradecen el apoyo financiero de los proyectos FDI 02C8PT-05 y Fondecyt 1050341. Además, agradecen las sugerencias de dos revisores anónimos que permitieron mejorar el presente manuscrito.

\section{REFERENCIAS}

Alcapán AC, RF Néspolo, JE Toro. 2007. Heritability of body size in the Chilean blue mussel (Mytilus chilensis Hupé 1854): effects of environment and aging. Aquac Res 38, 313-320.

Alvarez G, C Zapata, R Amaro, A Guerra. 1989. Multilocus heterozygosity at protein loci and fitness in the European oyster Ostrea edulis L. Heredity 63, 359-372.

Beaumont AR, MD Budd. 1983. Effects of self-fertilisation and other factors on the early development of the scallop Pecten maximus. Mar Biol 76, 285-289.

Blot M, C Thiriot-Quievreux. 1988. Multiple locus fitness in a transfer of adult Mytilus desolationis (Mollusca, Bivalvia). $23^{\text {rd }}$ European Marine Biology Symposium, University of Wales, UK, Pp 259-264.

David P. 1998. Heterozygosity-fitness correlations: a new perspectives on old problem. Heredity 80, 531-537.

Falconer DS, T Mackay. 1996. Introduction to quantitative genetics. $4^{\text {th }}$ ed. Longman Group Limited, London, UK.

Feráld J. 2002. How useful are the genetic markers in attempts to understand and manage marine biodiversity? J Exp Mar Biol Ecol 268, 121-145.

Gentili MR, AR Beaumont. 1988. Environmental stress, heterozygosity, and growth rate in Mitylus edulis L. J Exp Mar Biol Ecol 120,145-153.

Ibarra AM, P Cruz, B Romero. 1995. Effects of inbreeding on growth and survival of self-fertilized catarina scallop larvae, Argopecten circularis. Aquaculture 134, 37-47.

Ibarra AM, JL Ramírez, CA Ruiz, P Cruz, S Avila. 1999. Realized heritabilities and genetic correlation after dual selection for total weight and shell width in catarina scallop (Argopecten ventricosus). Aquaculture 175, 227-241.

Illanes JE. 1998. Experiencias de captación de larvas de ostión (Argopecten purpuratus) en Chile. En: Uribe E (ed). Producción de larvas y juveniles de especies marinas. Editorial Universitaria, Santiago, Chile, Pp 53-57.

Koehn RK, SE Shumway. 1982. A genetic/physiological explanation for differential growth rate among individuals of the American oyster, Crassostrea virginica (Gmelin). Mar Biol Lett 3, 35-42.
Liu ZJ, JF Cordes. 2004. DNA markers technologies and their applications in aquaculture genetics. Aquaculture 238, 1-37.

Martínez V. 2007. Using marker data in conventional breeding programmes: A case of study in selective breeding programs of scallops. Aquaculture 272, 287.

Martínez V, S Di Giovanni. 2007. Breeding programmes of scallops: Effect of self-fertilization when estimating genetic parameters. Aquaculture 272, 287.

Martínez V. 2008. Further insights of the variante component method for detecting dominant QTL in livestock and aquacultural species. Genet Sel Evol 40, 585-606.

Montoya M. 2005. Estudios genéticos moleculares y cuantitativos de la autofecundación en el ostión del norte Argopecten purpuratus (Lamarck, 1819). Tesis de Magíster, Facultad de Ciencias, Universidad Austral de Chile, Valdivia, Chile.

Rego I, A Martínez, A González-Tizón, J Veites, F Leiva, J Méndez. 2002. PCR technique for identification of mussel species. J Agr Food Chem 50, 1780-1784.

Rodhouse PG, JH McDonald, TIR Newell, RK Koehn. 1986. Gamete production, somatic growth and multiple-locus enzyme heterozygosity in Mytilus edulis. Mar Biol 90, 209-214.

SERNAPESCA, Servicio Nacional de Pesca. 2007. Anuario estadístico de pesca. Ministerio de Economía Fomento y Reconstrucción, Santiago, Chile.

Taud S, S Palacios. 2003. La acuicultura en Chile. Technopress SA, Salmon Chile.

Toro JE 1990. Respuesta a la selección, heredabilidades y correlación genética para los caracteres peso vivo y longitud de la concha en la Ostra europea Ostrea edulis Linne. Rev Biol Mar Oceanogr 25, 135-146.

Toro JE, PR Águila. 1995. Cruzamientos factoriales con cinco poblaciones de ostra chilena (Ostrea chilensis Philippi 1845) aisladas geográficamente. Arch Med Vet 27, 69-80.

Toro JE, AM Vergara, R. Galleguillos. 1996. Multiple-locus heterozygosity and physiology of the growth in different stages of the life cycle of the Chilean oyster Ostrea chilensis Philippi 1845. Mar Ecol PS 134, 151-158.

Toro JE, AM Vergara. 1998. Growth and heterozygosity in a 12-monthold cohort of Ostrea chilensis (Philippi, 1845) obtained by mass spawning in the laboratory. Mar Ecol PSZN 19, 311-323.

Toro JE, AC Alcapán, AM Vergara, JA Ojeda. 2004․ Heritability estimates of larval and spat shell height in the Chilean blue mussel (Mytilus chilensis Hupe 1854) produced under controlled laboratory conditions. Aquac Res 35, 56-61.

Toro JE, AC Alcapán, JA Ojeda, AM Vergara. 2004 . Selection response for growth rate (shell height and live weight) in the Chilean blue mussel (Mytilus chilensis Hupe 1854). J Shellfish Res 23, 753-757.

Tuan R, LCG Simões. 1998. Effect of self-fertilization on Biomphalaria tenagophila (Orbigny, 1835) (Pulmonata: Planorbidae). Genet Mol Biol 21, 1415-1457.

Vernon JG, C Jones, LR Noble. 1995. Random amplified polymorphic DNA (RAPD) markers reveal cross-fertilisation in Biomphalaria glabrata (Pulmonata: Basommatophora). J Mollus Stud 61, 455-465.

Winkler FM, B Estévez. 2003. Effects of self-fertilization on growth and survival of larvae and juveniles of the scallop Argopecten purpuratus L. J Exp Mar Biol Ecol 292, 93-102. 